\title{
The landscape of genetic variation in dilated cardiomyopathy as surveyed by clinical DNA sequencing
}

\author{
Trevor J. Pugh, PhD1,2,3, Melissa A. Kelly, MS1, Sivakumar Gowrisankar, PhD1, Elizabeth Hynes, BA ${ }^{1}$, \\ Michael A. Seidman, MD, PhD¹,3, Samantha M. Baxter, MS¹, Mark Bowser, MS ${ }^{1}$, Bryan Harrison, BA ${ }^{1}$, \\ Daniel Aaron, BA ${ }^{1}$, Lisa M. Mahanta, BA ${ }^{1}$, Neal K. Lakdawala, MD4, Gregory McDermott, BA ${ }^{1}$, \\ Emily T. White, MS ${ }^{1}$, Heidi L. Rehm, $\mathrm{PhD}^{1,3}$, Matthew Lebo, $\mathrm{PhD}^{1,3}$ and Birgit H. Funke, PhD ${ }^{1,2}$
}

\begin{abstract}
Purpose: Dilated cardiomyopathy is characterized by substantial locus, allelic, and clinical heterogeneity that necessitates testing of many genes across clinically overlapping diseases. Few studies have sequenced sufficient individuals; thus, the contributions of individual genes and the pathogenic variant spectrum are still poorly defined. We analyzed 766 dilated cardiomyopathy patients tested over 5 years in our molecular diagnostics laboratory.

Methods: Patients were tested using gene panels of increasing size from 5 to 46 genes, including 121 cases tested with a multiple-cardiomyopathy next-generation panel covering 46 genes. All variants were reassessed using our current clinical-grade scoring system to eliminate false-positive disease associations that afflict many older analyses.
\end{abstract}

Results: Up to $37 \%$ of dilated cardiomyopathy cases carry a clinically relevant variant in one of 20 genes, titin (TTN) being the largest contributor (up to $14 \%$ ). Desmoplakin (DSP), an arrhythmogenic right ventricular cardiomyopathy gene, contributed $2.4 \%$, illustrating the utility of multidisease testing. The clinical sensitivity increased from 10 to $37 \%$ as gene panel sizes increased. However, the number of inconclusive cases also increased from 4.6 to $51 \%$.

Conclusion: Our data illustrate the utility of broad gene panels for genetically and clinically heterogeneous diseases but also highlight challenges as molecular diagnostics moves toward genome-wide testing.

Genet Med advance online publication 6 February 2014

Key Words: cardiomyopathy; genetic heterogeneity; molecular diagnostics; next-generation sequencing; variants
Dilated cardiomyopathy (DCM) is a frequent cause of heart failure and sudden cardiac death worldwide. ${ }^{1}$ Initially believed to be largely secondary to environmental causes, it is now well established to have a significant genetic etiology. ${ }^{2}$ Genetic heterogeneity is more pronounced than in other cardiomyopathies, with currently more than 40 DCM genes implicated, most contributing only a small or modest fraction to the pathogenic variation in DCM patients. ${ }^{3-5}$ Clinical overlap with other cardiomyopathies (hypertrophic cardiomyopathy (HCM), arrhythmogenic right ventricular cardiomyopathy (ARVC)) has been described ${ }^{6-10}$ and can lead to diagnostic uncertainty in some cases. Sequencing a wider array of genes can therefore be beneficial but was impossible until recently, when next-generation sequencing (NGS) technologies enabled truly comprehensive testing. ${ }^{11-13}$

NGS also enabled broad surveys of genetic variation present in the general population, contributing to a better ability to distinguish between pathogenic variants and low-level population variation in patients. Exome-wide sequencing data, such as those from the National Heart, Lung, and Blood Institute Exome Sequencing Project (NHLBI ESP) ${ }^{14}$ or the 1000 Genomes Project, ${ }^{15}$ are now publicly available. Analysis of these data has led to the realization that many variants originally believed to be disease causing based on their absence in small- or moderately-sized control cohorts are more likely moderately rare, benign variants. ${ }^{16,17}$ Existing inaccurate variant-disease associations pose a challenge for clinical variant interpretation and indicate a critical need for an iterative, systematic reassessment of previously classified genetic variation.

We report here the diagnostic use of our custom targeted NGS panel for inherited cardiomyopathies. We present a comparison of this test's clinical detection rate with those of predecessor tests based on array-based and Sanger sequencing technologies, as well as the results of a systematic reevaluation of all variants we detected during 5.5 years of DCM testing. This analysis represents one of the largest cohorts of DCM patients clinically sequenced to date. Combined with our stringent reassessment of variants, it refines gene-specific detection rates and the spectrum of pathogenic variants in DCM. We also confirm previous reports that pathogenic variants in genes not traditionally associated with DCM are present in a significant number of patients, underscoring the utility of multiple-disease panel testing. 


\section{MATERIALS AND METHODS}

\section{Molecular diagnostic tests for cardiomyopathy}

Five molecular testing panels were used by our laboratory during the time period of our study: two Sanger sequencing panels together targeting 10 genes ("Sanger"), a microarray-based sequencing assay targeting 19 genes ("DCM CardioChip"), and two NGS assays targeting 24 DCM genes ("DCM panel") or 46 genes associated with DCM, HCM, ARVC, left ventricular noncompaction (LVNC), restrictive cardiomyopathy (RCM), and catecholaminergic polymorphic ventricular tachycardia (CPVT; "Pan Cardiomyopathy Panel"). See Supplementary Data online for gene lists. The evidence linking these genes to cardiomyopathy has been published elsewhere. ${ }^{3}$ The development of the Sanger sequencing and microarray-based assays has been previously described. ${ }^{18}$ The development and clinical performance characteristics of our NGS-based Pan Cardiomyopathy Panel are described in the Supplementary Data and Supplementary Table S6 online. In total, we analyzed 766 DCM cases using Sanger sequencing (176 cases), the DCM CardioChip microarray (417), NGS-based gene panels (149), or a combination of these (24). Patients who were tested more than once were counted toward the larger panel. For microarray and NGS tests, Sanger sequencing was used to fill in failed bases and to confirm all novel or clinically relevant variants (classified as being of "unknown significance," "likely pathogenic," or "pathogenic").

\section{Sequencing technologies}

Methods used for polymerase chain reaction, Sanger sequencing, and array-based sequencing have been previously described. ${ }^{18}$ For NGS, bar coded libraries for 10 samples were pooled before target capture (Agilent SureSelect) and sequenced on an Illumina HiSeq2000 instrument (50 base paired end mode). Reads were aligned using $\mathrm{BWA}^{19}$ and recalibrated and realigned using GATK, version 1.0.4705. ${ }^{20}$ Unified Genotyper was used to detect single-nucleotide variants, and IndelGenotyper was used to detect insertions and deletions (in/dels). ${ }^{21}$ Variants were annotated and filtered using a custom script that queried our clinical-grade variant database. ${ }^{22}$

\section{Variant interpretation criteria}

Variants were classified as shown in Table 1. Major drivers include the frequency and number of alleles in patient and control populations, degree of segregation with disease, functional evidence, predicted protein effect, and comparison with the established spectrum of pathogenic variation in a gene. Variants of unknown significance (VUSs) whose evidence level borders on but is not quite sufficient to be classified as "likely pathogenic" were classified as "VUS-favor pathogenic."

\section{Mapping of titin variants}

We compared titin (TTN) variants from our cohort and from cases published by Herman et al..$^{23}$ ("probands") with those detected in $\sim 6,500$ individuals sequenced by ESP (downloaded 21 January 2013, "controls"). The observed distribution of variants across the gene was compared with the expected frequencies for the same gene using a binomial test with expected frequencies determined by the fraction of the overall sequence represented by each band. This analysis was performed independently for probands and controls. A Fisher exact test was used to compare the distribution of variants in probands with the distribution of variants in controls. Bonferroni correction was performed on all analyses to allow for multiple testing. Odds ratios for probands versus controls were calculated, and confidence intervals were determined using the conditional maximum likelihood/Fisher method. All statistical analyses were performed using the R software package.

\section{Patient cohort}

\section{RESULTS}

Our patient cohort represents a broad referral population of 766 individuals with DCM or clinical features consistent with DCM, based on medical and family history information provided by ordering providers. This study was approved by the Partners HealthCare Institutional Review Board. We excluded cases with confirmed diagnoses of any other type of cardiomyopathy, other structural heart disease or congenital heart disease, or syndromic or environmental causes. Fourteen cases had clinical features or a family history of skeletal myopathy or muscular dystrophy, in addition to a clinical diagnosis or clinical features of DCM, and were included because muscle disease is within the phenotypic spectrum for several genes we tested. This cohort was predominantly white (464 were white, 86 were black or African American, 43 were Hispanic or Latino, 22 were Asian, 7 were Ashkenazi Jews, 22 were of mixed ancestry, and 122 were of unspecified ancestry). The age composition is unusual in that $37 \%$ of patients were younger than 18 years $(n=286)$ with a high number of infants (138 cases $\leq 2$ years of age; Supplementary Figure S1 online). Our cohort had slightly more males than females, 53\% vs. $43 \%$ (404 males vs. 330 females, 32 unspecified). A family history (defined as a report of DCM or clinical features of DCM, nonspecific cardiomyopathy, sudden death, or heart failure) was indicated in 425 (55\%) cases. All patients received diagnostic gene panel testing of 5-46 genes between 2007 and 2012. As a result of the evolution in testing over time, genes on the oldest panels have been tested in nearly every case, whereas new genes such as TTN have been tested in less than $25 \%$ of cases.

\section{Variant assessment and result interpretation}

Across all 766 DCM cases (Supplementary Table S1 online), we detected 893 unique variants, not counting common variants classified as "benign" (Supplementary Table S3 online).

Patients were tested over a range of 5.5 years, and during this time the medical genetics community has undergone a significant evolution in how sequence variants are interpreted. Our most recent variant classification rules were implemented in the fall of 2011. Therefore, to ensure consistency of variant interpretation across our cohort, we performed a stringent clinical reassessment of 275 variants classified before that date using rules summarized in Table 1. Counts of variants assigned to each category before and after this reassessment are tabulated in Table 2. Regarding variants previously classified 
Table 1 Clinical classification criteria

\begin{tabular}{|c|c|c|c|}
\hline \multicolumn{4}{|c|}{ Clinical-grade variant classification criteria } \\
\hline \multirow[t]{4}{*}{ Pathogenic } & \multirow[t]{3}{*}{ Missense } & $\begin{array}{l}\text { Segregation of the } \\
\text { variant in at least five } \\
\text { affected family members }\end{array}$ & $\begin{array}{l}\text { Unaffected individuals are not included because all inherited cardiomyopathies are } \\
\text { afflicted by reduced penetrance and clinical variability }\end{array}$ \\
\hline & & $\begin{array}{l}\text { Strong evolutionary } \\
\text { conservation of the } \\
\text { affected amino acid }\end{array}$ & \multirow{2}{*}{$\begin{array}{l}\text { Presence of a variant in the general population (if rare) requires a higher number of } \\
\text { segregations (at least } 10 \text { affected family members) }\end{array}$} \\
\hline & & $\begin{array}{l}\text { Absence from large, race- } \\
\text { matched populations } \\
\text { (e.g., ESP cohorts) }\end{array}$ & \\
\hline & Other & \multicolumn{2}{|c|}{$\begin{array}{l}\text { Truncating (nonsense, frameshift) and splice variants affecting the invariant } \pm 1,2 \text { positions are classified as pathogenic } \\
\text { provided that both criteria are met: (i) the type of variant is prevalent among known pathogenic variants for a particular gene; } \\
\text { (ii) the variant is not located distal to the terminal } 50 \mathrm{bp} \text { of the penultimate exon, where nonsense-mediated decay is unlikely }\end{array}$} \\
\hline \multirow[t]{5}{*}{$\begin{array}{l}\text { Likely } \\
\text { pathogenic }\end{array}$} & Missense & $\begin{array}{l}\text { Segregation of the } \\
\text { variant in three to four } \\
\text { affected family members }\end{array}$ & $\begin{array}{l}\text { For variants that are present in the general population at low frequency, a higher } \\
\text { threshold of segregation is required ( } 5-9 \text { segregations) }\end{array}$ \\
\hline & & AND & \multirow{4}{*}{$\begin{array}{l}\text { Strong functional evidence (from animal models) or strong established clinical } \\
\text { correlation of the gene with the patient's phenotype can substitute for segregation data }\end{array}$} \\
\hline & & $\begin{array}{l}\text { Strong evolutionary } \\
\text { conservation of the } \\
\text { affected amino acid }\end{array}$ & \\
\hline & & AND & \\
\hline & & $\begin{array}{l}\text { Absence from large, } \\
\text { race-matched populations } \\
\text { (e.g., ESP cohorts) }\end{array}$ & \\
\hline VUS & & \multicolumn{2}{|c|}{$\begin{array}{l}\text { This category includes all other variants with insufficient evidence to be classified as above, that do not meet criteria for likely } \\
\text { benign or benign (below), or that have conflicting data }\end{array}$} \\
\hline $\begin{array}{l}\text { (Likely) } \\
\text { Benign }\end{array}$ & & \multicolumn{2}{|c|}{$\begin{array}{l}\text { These variants have MAFs exceeding set thresholds for likely benign (MAF }>0.3 \% \text { ) or benign (MAF }>1 \% \text { ), provided the } \\
\text { cohort was of sufficient size to provide confidence in the accuracy of the frequency. For smaller cohort sizes, a higher } \\
\text { threshold was required (likely benign: } 1 \% \text {, benign: } 3 \% \text { ) }\end{array}$} \\
\hline
\end{tabular}

ESP, Exome Sequencing Project; MAF, minor allele frequency; VUS, variant of unknown significance.

as of unknown significance, 5 of $106(4.7 \%)$ were reclassified as likely pathogenic and 6 were downgraded to likely benign. Importantly, this reanalysis resulted in downgrading 12 of 56 (21\%) likely pathogenic variants to VUSs $(n=10)$ or likely benign $(n=2)$, which was largely driven by their presence in large populations sequenced by the NHLBI ESP ${ }^{14}$ and the 1000 Genomes ${ }^{15}$ projects. This prevalence of historically misclassified pathogenic variation is consistent with a recent systematic reanalysis of putatively pathogenic cardiomyopathy variants in the NHLBI ESP cohort. ${ }^{24}$ Of the 10 variants originally classified as "pathogenic," only one was downgraded to "likely pathogenic."
Table 2 Reinterpretation of previously classified variants

\begin{tabular}{|l|c|c|c|c|c|}
\hline \multirow{2}{*}{} & & \multicolumn{4}{|c|}{ After reclassification } \\
\cline { 2 - 6 } & & $\mathrm{P}$ & LP & VUS & LB \\
\hline \multirow{3}{*}{ Original } & $\mathrm{P}$ & $\mathbf{9}$ & 1 & 0 & 0 \\
\cline { 2 - 6 } & LP & 1 & $\mathbf{4 3}$ & 10 & 2 \\
\cline { 2 - 6 } & VUS & 0 & 5 & $\mathbf{9 5}$ & 6 \\
\cline { 2 - 6 } & LB & 0 & 0 & 0 & $\mathbf{1 0 3}$ \\
\hline
\end{tabular}

Bordered boxes indicate the number of variants that did not change classification and shaded boxes indicate variant reclassifications that change the reported result (i.e., positive becomes negative/inconclusive, or negative/inconclusive becomes positive).

LB, likely benign; LP, likely pathogenic; P, pathogenic; VUS, variant of unknown significance. 
The resultant number of variants assigned to each category was as follows: 376 "likely benign," 374 of "unknown significance," 43 "VUS-favor pathogenic," 87 "likely pathogenic," and 13 "pathogenic" (Supplementary Table S3 online). The vast majority of all variants (84\%) were found in a single patient. More singleton variants were found among variants classified as of "unknown significance" or higher (90\%) as compared with those classified as "likely benign" (77\%).

\section{Clinical sensitivity}

To define lower and upper estimates of clinical sensitivity, we used two thresholds to identify "positive" cases (those that contain variants of known, likely, or strongly suspected clinical significance). The "lower bound" considered only cases with likely pathogenic and pathogenic variants. Our earlier analysis predicted that nearly half of all VUSs may be pathogenic. ${ }^{25}$ Therefore, our "upper bound" approach included additional cases with the most convincing VUSs (VUS-favor pathogenic; Supplementary Table S3 online). As gene panel sizes increased from 5 to 46 genes, the clinical sensitivity for DCM in our laboratory more than tripled, from a range of $7.7-10 \%$ (lower bound-upper bound) to a range of 27-37\% (Figure 1). However, this improvement is tempered by an emerging interpretive challenge because the percentage of patients receiving an inconclusive test result (only VUSs detected) increased from $4.6-6.5 \%$ to $51-61 \%$. These increases were driven largely by the inclusion of TTN, which encodes the largest human protein.

The presence of a family history is often indicative of an underlying genetic etiology. Interestingly, the clinical sensitivity for individuals with a family history of DCM was similar to that obtained for the entire cohort (with positive rates improving from $6.9-8.6 \%$ to $31-38 \%$ ) and is consistent with our previous, smaller study. ${ }^{26}$ This is different from what has been reported for HCM, for which a family history substantially increases the likelihood of detecting a clinically relevant variant. A second variable that is often suspected to correlate with detection rates is age. Our previous analyses indicated that the overall detection rate for pathogenic DCM variants across all genes tested did not differ among age groups. ${ }^{26}$ In our current data set, detection rates did not differ among age groups except for those for the two most recent gene panels ( 24 and 46 genes; Supplementary Figure S2 online). Here, the higher detection rate in the adult and pediatric groups was driven mostly by $T T N$ and $D S P$, although it should be noted that the numbers behind some of these detection rates are small (for confidence intervals, see Supplementary Table S2 online).

\section{Detection rates and variant spectra by gene}

To determine the contribution of each gene, we counted the number of positive tests (those containing variants of known, likely, or strongly suspected clinical significance as defined above) for each gene individually (Figure 2, Supplementary Table S2 online). This was done for the entire cohort but also separately for each age group. The spectrum of variant types underlying the detection rates is depicted in Supplementary Figure S3 online.

Overall, TTN was the largest contributor of positive test results (12-14\%), largely due to truncating variants or variants

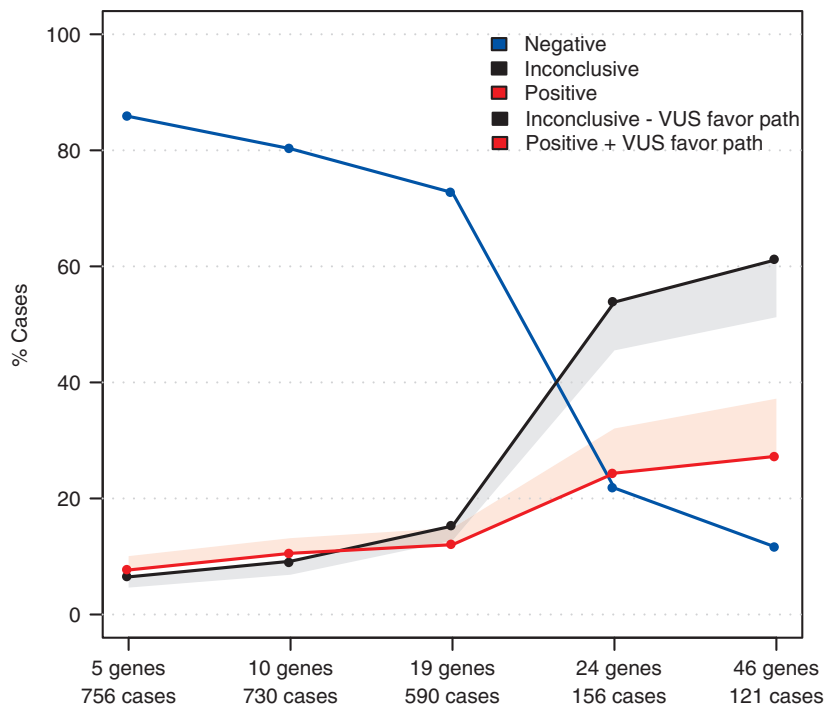

Figure 1 Increase in detection rate with expanded gene panels is tempered by an even larger increase in the number of inconclusive reports. The total detection rate for increasing numbers of DCM genes tested from 2007 to 2011 is shown. For each set, the percentages are calculated as the number of probands with a given result (positive, negative, or inconclusive) divided by the total number of tests using that gene set. The solid red line represents the lower bound of the clinical sensitivity (cases containing variants of established or likely pathogenicity); the shaded red area delineates the "upper bound" of the clinical sensitivity and includes cases with a variant classified as variant of unknown significance (VUS)-favor pathogenic. The solid black line represents the upper bound for the fraction of inconclusive cases (including those bordering on a likely pathogenic classification), and the shaded gray area represents the lower bound. The solid blue line represents the percentage of negative cases over time. 
a

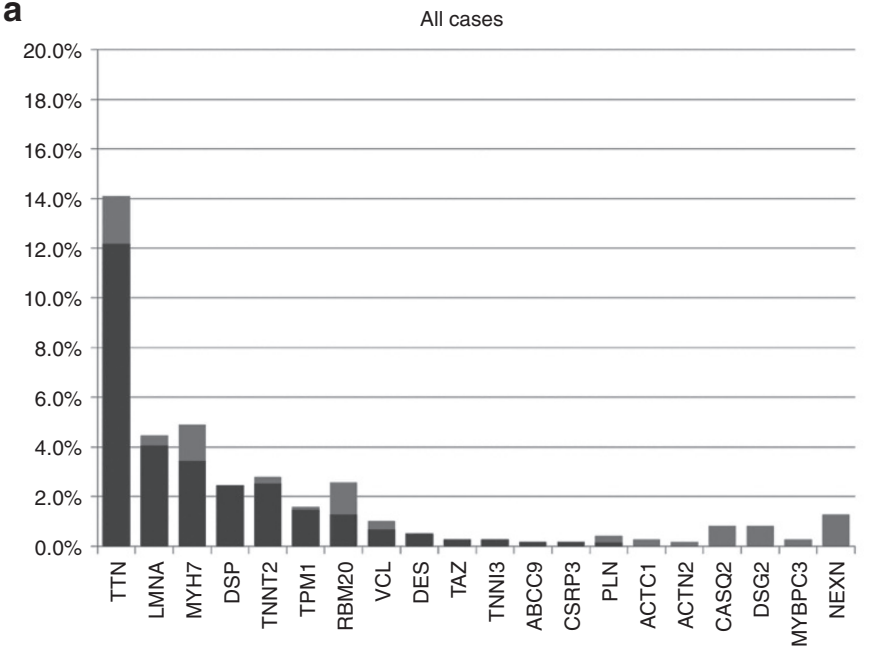

C

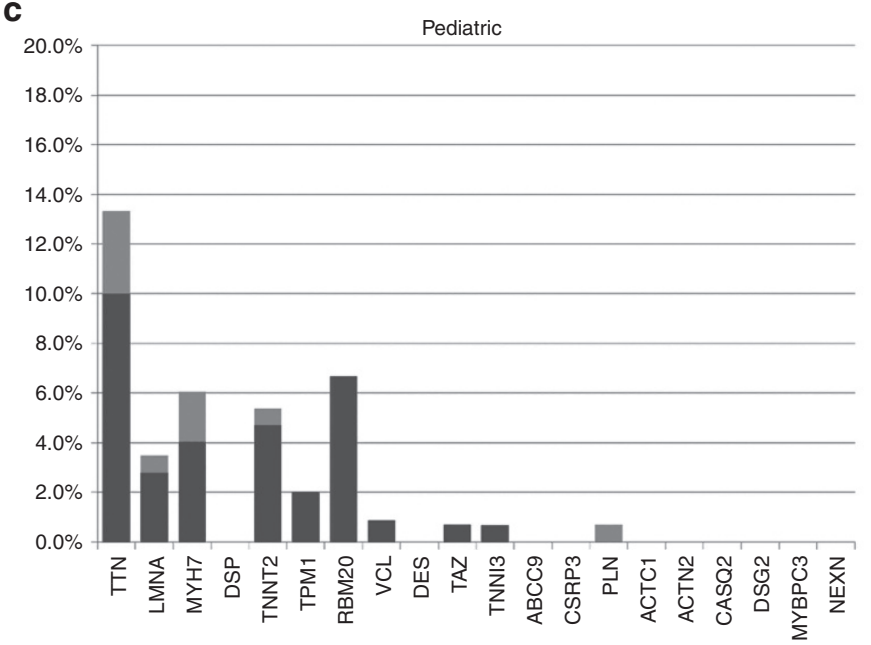

b

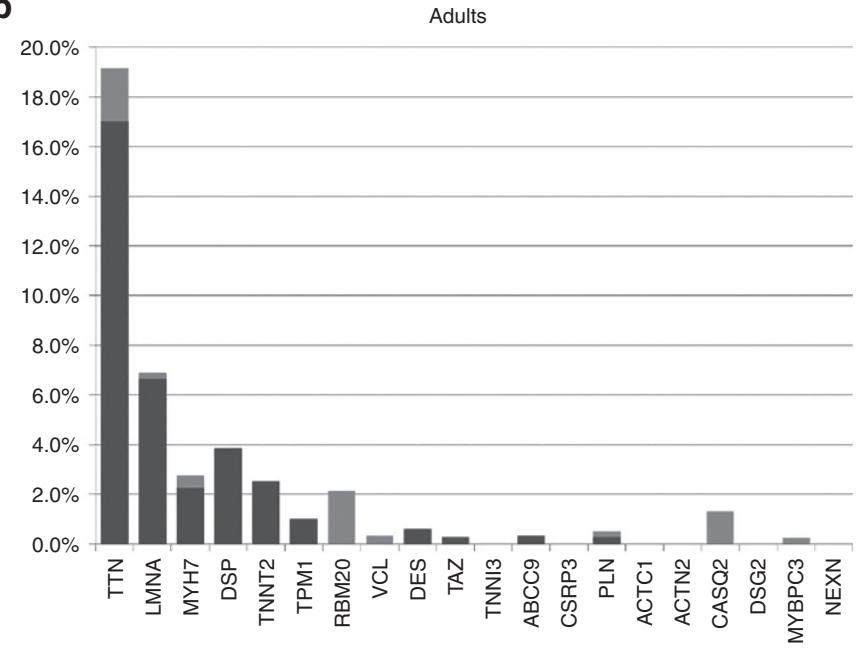

d

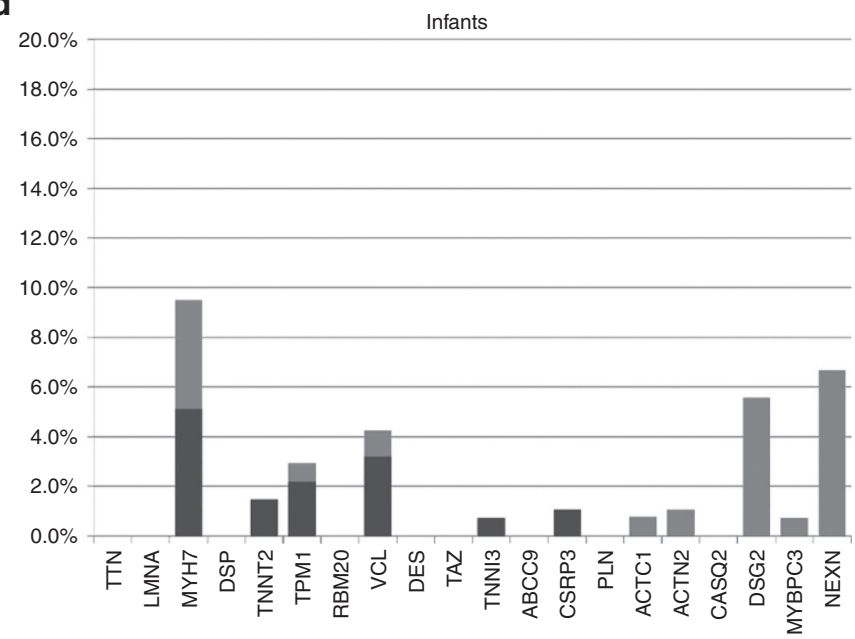

Figure 2 Detection rate by gene. Genes are displayed on the $x$ axis in decreasing order. Dark gray portions represent the percentage of cases with a likely pathogenic or pathogenic variant in a gene, and light gray portions represent the percentage of cases that harbored a variant bordering on but not formally classified as "likely pathogenic" (variant of unknown significance (VUS)-favor pathogenic). (a) Detection rates by gene for all cases. (b) Detection rates by gene for all adults (aged 18 years or older). (c) Detection rates by gene for all pediatric cases (aged 2 to $<18$ years). (d) Detection rates by gene for all infants (0 to $<2$ years).

altering the conserved splice consensus sequence as previously reported. ${ }^{23}$ We identified 152 missense TTN VUSs that drove a surge of the above-mentioned inconclusive test results (Figure 1). The second highest contributor was LMNA (4.1-4.5\%), followed by $M Y H 7$ (3.4-4.9\%). Clinically significant variants in DSP, an ARVC gene, were found in $2.4 \%$ of cases, consistent with previous reports, ${ }^{1}$ which illustrates the utility of broad testing.

The spectrum of contributing genes differed among the age groups, most prominently when comparing the infant group with the pediatric and adult groups. Some of these differences are already established (e.g., the finding that $L M N A$ variants are more likely to present in adulthood); some have not yet been described but will need to be confirmed by other studies (e.g., the findings that DSP variants were unique to the adult cohort and $R B M 20$ variants were enriched in the pediatric group).

We did not detect any variants classified as "pathogenic," "likely pathogenic," or "VUS-favor pathogenic" in 24 of 46 genes tested. For some, this was expected because there is currently no reported association with DCM. However, 11 of these 24 genes (ANKRD1, CAV3, CRYAB, CTF1, DSC2, EMD, FHL2, LAMA4, LAMP2, MYH6, and PKP2) have been previously reported as likely or candidate DCM genes. ${ }^{3}$

Of particular interest is the MYBPC3 gene, for which published detection rates range from 1 to $10 \% .{ }^{18}$ We failed to detect any pathogenic or likely pathogenic variants in this gene. Only three MYBPC3 variants were of possible clinical significance (VUS-favor pathogenic). Closer examination suggested that two of them may be HCM variants (p.Asp605Asn and p.Ile659Thr; for detailed interpretations, see Supplementary Data online).

\section{Distribution of TTN-truncating variants in individuals with DCM and in the general population}

From an analysis of 312 DCM patients and 249 controls, Herman et al. ${ }^{23}$ reported a clustering of truncating variants in the A-band 
of TTN. ${ }^{23}$ To validate and expand on this finding, we combined the variants from Herman et al. ${ }^{23}(n=55)$ with TTN -truncating variants detected in our study $(n=18)$. The NHLBI ESP cohorts ( 6,500 individuals of European or African-American ancestry) served as a control cohort, representing the general population. Truncating variants in the large exon unique to the Novex-3 transcript were excluded because the biological significance of this splice form is not well understood. Supplementary Table S4 online lists all TTN-truncating variants used for this analysis.

The control set contained 43 truncating variants identified in 107 individuals. Assuming that no individual carried more than one truncating variant, this corresponds to a frequency of 107 per 6,500 individuals (1.65\%). The TTN A-band was enriched for truncating variants in DCM probands as compared with controls (odds ratio $=14.6 ; 99 \%$ confidence interval: $\left.4.32-58.5 ; P<2 \times 10^{-10}\right)$. The frequency in controls as well as the clustering of variants in the A-band in probands is consistent with results reported by Herman et al. ${ }^{23}$ In addition, our data showed a reduced frequency of variants in the I-band in probands as compared with controls (odds ratio $=0.13 ; 99 \%$ confidence interval: $\left.0.04-0.43 ; P<2 \times 10^{-6}\right)$. No difference in frequency was detected in the Z- and M-bands (Figure 3).

\section{Loss-of-function variants in vinculin (VCL) as an emerging rare cause of DCM}

Many DCM genes contribute only a very small fraction of disease-causing variants, making it difficult for any individual study to establish the pathogenic variant spectrum. One such gene is the VCL gene, which encodes a Z-disk protein and was initially implicated in the etiology of cardiomyopathy based on an in-frame deletion (p.Leu955del) detected in a single DCM patient. ${ }^{27}$ Our reassessment revealed that this variant is present in $0.4 \%(17 / 4,264)$ of African-American chromosomes sequenced by the NHLBI ESP project, suggesting (although not proving) that it may be benign. Two additional missense variants have been reported in HCM patients, p.Leu277Met ${ }^{28}$ and p.Arg975Trp 29 ; the latter has also reported in a DCM patient. ${ }^{27}$ According to our criteria, both variants were classified as "VUSs," therefore leaving the significance of all previously published variants uncertain. In our cohort (VCL tested in 590 patients), we detected four heterozygous variants that probably lead to loss of protein function: p.Arg105X, p.Arg188X,

\begin{tabular}{lc|c|c|c} 
& & & \\
Controls & $8.5 \%$ & $61.7 \%$ & $19.2 \%$ & $10.6 \%$ \\
Probands & $2.7 \%$ & $17.8 \%$ & $78.1 \%$ & $1.4 \%$ \\
TTN domains & $\mathrm{Z}$ & $\mathrm{I}$ & $\mathrm{A}$ & $\mathrm{M}$ \\
& & $P<2 \times 10^{-6}$ & $P<2 \times 10^{-10}$ & \\
& & 0.13 & 14.6 & \\
Odds ratio & & $(99 \% \mathrm{Cl}=0.04-0.43)$ & $(99 \% \mathrm{Cl}=4.32-58.5)$ &
\end{tabular}

Figure 3 Positional distribution of titin (TTN) variants. Frequencies of variants in the I-band and the A-band in probands and controls, as well as the $P$ value for probands versus controls and the odds ratio of finding variants in the respective bands in probands versus controls. $\mathrm{Cl}$, confidence interval.
p.Asn220LysfsX21, and p.Arg547X. This is the first report of loss-of-function VCL variants in DCM patients and is consistent with mouse studies that suggest that loss of VCL may lead to DCM. ${ }^{30}$ It does not, however, refute the possibility that other variant types in this gene can lead to disease. Of note, loss-of-function $V C L$ variants appear to be rare in the general population because only three such alleles (p.Asn220LysfsX21, p.Ala573HisfsX8, and p.Arg409X) have been found, each as singletons, in more than 8,250 chromosomes examined by the NHLBI ESP (accessed 13 October 2013).

\section{Contribution of desmosomal gene variants to DCM}

Two studies have sequenced desmosomal genes (DSP, DSG, DSC2, PKP2, and JUP), which have been associated with ARVC, in patients with DCM. ${ }^{10,31}$ These studies reported disease-causing variants in $5 \%$ and $13 \%$ of cases. Several of these variants have also been detected by our laboratory but are classified as likely benign or benign based on their frequency in the NHLBI ESP cohorts. Removing these variants, the detection rates in these two cohorts decrease to $3 \%$ and $7.9 \%$, largely due to variants in the DSP $(n=7)$ and DSG2 $(n=2)$ genes (Supplementary Table S5 online). This is consistent with the results from our cohort, for which the same two desmosomal genes contributed to positive test results (DSP: $3 / 123$ cases $=2.4 \%, D S G 2: 1 / 123$ cases $=0.8 \%$ ).

\section{DISCUSSION}

Owing to locus, allelic, and clinical heterogeneity, comprehensive testing for DCM benefits from sequencing the entire coding region of a growing number of genes. Most studies available to date are limited in the number of genes and/or the number of individuals sequenced and, consequently, estimates of detection rates are not very robust. In addition, clinical variant classification has greatly evolved over the past decade, and new knowledge has revealed that many past variant-disease associations are flawed.

\section{Impact of NGS panels on medical sequencing}

Genetic testing panels have undergone a dramatic expansion in recent years. For DCM, our test panels have increased from only 5 genes (MHY7, MYBPC3, TNNT2, TNNI3, and TPM1) to 46 covering DCM genes as well as genes involved in other cardiomyopathies (HCM, ARVC, LVNC, and RCM). The most prominent addition is the TTN gene, which was virtually untestable in the clinic before the advent of NGS technologies and has the highest detection rate among all known DCM genes. NGS has also enabled clinical diagnostic laboratories to configure gene panels that encompass multiple overlapping clinical entities, which is beginning to change how genetic testing is used in clinical medicine. Traditionally used to confirm or rule out a clinical diagnosis, genetic testing is now increasingly part of the diagnostic process. Among the inherited cardiomyopathies, this benefit is probably most pronounced for DCM because it can be an end-stage presentation of HCM in a minority of cases ${ }^{6}$ and has some clinical overlap with ARVC. ${ }^{7,8}$ Our analysis confirmed earlier reports that pathogenic variation in desmosomal 
genes contributes a portion of pathogenic variation in DCM patients. It remains to be shown whether such cases represent misdiagnosed ARVC or whether desmosomal variants can cause both disorders.

\section{Detection rate and importance of continuous reanalysis of variants}

Among the inherited cardiomyopathies, the benefit of expanded gene testing is most pronounced for DCM because a large number of genes (22 in our study) harbor clinically significant variants causative of this disease. ${ }^{1,5}$ This is in stark contrast to $\mathrm{HCM}$, in which $\sim 80 \%$ of pathogenic variation is contributed by only two genes, MYH7 and MYBPC3. ${ }^{3}$ With clinically significant variants present in up to $37 \%$ of individuals with DCM and up to $38 \%$ of individuals with familial DCM, our detection rates are lower than those expected based on previous meta-analyses of published detection rates. ${ }^{5,18}$ This is probably caused by a combination of factors, including those listed below (see Study Limitations). An emerging major reason for lower detection rates is that many past studies classified variants as pathogenic based on insufficient data that led to incorrect variant-disease associations. ${ }^{16,17}$ In our study, 9\% (25/275) of variants underwent a change in classification. Importantly, nearly half (12) of these changes were substantial in that they affected "likely pathogenic" variants that were downgraded to "VUS" ( $n$ $=10)$ or likely benign $(n=2)$, largely due to their identification in large cohorts representing the general population. This type of classification change is of high significance in a clinical setting because it changes the overall result communicated to the patient from "positive" (a likely or definitive cause for the patient's disease was identified) to "inconclusive" or "negative." Such changes can dramatically alter medical management and thus underscore the critical need for conservative interpretation of variants and for reevaluating all disease-causing variants published before the availability of these resources.

\section{Reevaluating $M Y B P C 3$ as a DCM gene}

The MYBPC3 gene was first known for its major contribution to HCM and was therefore a logical candidate gene for DCM. A large number of studies have sequenced this gene, typically in small cohorts and have reported it as a DCM gene with various detection rates of up to $10 \%{ }^{18}$ We failed to detect any likely pathogenic or pathogenic variants in our cohort and suspect that two of the three variants that came close to a "likely pathogenic" classification are HCM variants (raising the possibility that these patients had end-stage HCM rather than primary DCM). Therefore, contrary to some published studies, our data suggest that $M Y B P C 3$ does not contribute significantly to primary DCM, although our cohort size is not large enough to confidently exclude it as a DCM gene.

\section{Clinical sensitivity and distribution of truncating variants in TTN}

In our study, TTN contributed up to $14 \%$ of all clinically significant cases, which is much lower than the detection rate reported by Herman et al. (up to 27\%). ${ }^{23}$ Multiple factors probably contributed to this difference. First, our cohort represents a broad referral population tested in our molecular diagnostic laboratory, which is different from a clinical study in that detailed clinical data supporting the indicated diagnosis are often not available. Second, Herman et al. ${ }^{23}$ used different variant classification criteria that were less stringent than ours (e.g., they included variants outside the highly conserved $\pm 1,2$ splice consensus in the absence of additional supporting data, which would be classified as "VUSs" using our rules). Consistent with Herman et al., ${ }^{23}$ we confirmed that TTN-truncating variants are present in the general population (1.65\% in the ESP cohort, $3 \%$ in the control cohort analyzed by Herman et al.). Our expanded analysis also confirmed that TTN-truncating variants appear to be enriched in the A-band in DCM cases as compared with the NHLBI ESP cohorts, which are used as a proxy for the general population. A novel observation was that truncating variants were significantly less frequent in the I-band in cases as compared with the ESP cohorts. It is reasonable to hypothesize that variants in the A-band have a higher likelihood of being pathogenic. The reduced frequency of truncating variants in the I-band in the patient cohort as compared with the ESP cohort increases the likelihood that these have a milder effect, although additional studies are needed to further investigate this. Nine of 43 variants present in the ESP cohort map to the A-band. In the absence of clinical and demographic information for the ESP cohort, it remains possible that these represent pathogenic variants in individuals who have not yet developed overt disease. This is not surprising because Herman et al. ${ }^{23}$ showed that TTN variants are highly penetrant but primarily after the age of 40 .

\section{Study cohort}

Our cohort is unusual in that only $\sim 50 \%$ of patients reported a family history and a high proportion of patients were infants and pediatric cases. This is in contrast to current guidelines (recommending testing mainly for patients with a family history) and beliefs (that genetic DCM is rare in infants). Our study revealed that the rate of clinically significant variants may be higher than previously thought, although this will need to be replicated by independent studies.

\section{Study limitations}

Our study relied on clinical data provided by ordering providers at the time of testing. In contrast to a controlled clinical study in which all patients are evaluated using a common set of diagnostic criteria, our clinical data are likely more heterogeneous.

A second limitation is that due to the expanding size of our test panels over time, not all genes were tested in all cases and therefore gene-specific detection rates are representative for the number of patients tested, rather than for the entire cohort.

Third, although our study is, to our knowledge, the largest reported analysis of sequence variation in DCM patients to date, it is not large enough to be immune to statistical fluctuations that commonly afflict small- to medium-sized cohorts 


\section{SUPPLEMENTARY MATERIAL}

Supplementary material is linked to the online version of the paper at http://www.nature.com/gim

\section{ACKNOWLEDGMENTS}

We thank the staff and fellows of the Laboratory for Molecular Medicine for their technical assistance in generating and interpreting these data.

\section{DISCLOSURE}

This work was funded by internal operating funds of the Partners HealthCare Center for Personalized Molecular Medicine. The Laboratory for Molecular Medicine is a nonprofit, fee-for-service laboratory offering testing for dilated cardiomyopathy. B.H.F. is a member of the scientific advisory board of InVitae. The other authors declare no conflict of interest.

\section{REFERENCES}

1. Lakdawala NK, Winterfield JR, Funke BH. Dilated cardiomyopathy. Circ Arrhythm Electrophysiol 2013;6:228-237.

2. Burkett EL, Hershberger RE. Clinical and genetic issues in familial dilated cardiomyopathy. J Am Coll Cardiol 2005;45:969-981.

3. Teekakirikul P, Kelly MA, Rehm HL, Lakdawala NK, Funke BH. Inherited cardiomyopathies: molecular genetics and clinical genetic testing in the postgenomic era. J Mol Diagn 2013;15:158-170.

4. Ackerman MJ, Priori SG, Willems S, et al.; Heart Rhythm Society (HRS); European Heart Rhythm Association (EHRA). HRS/EHRA expert consensus statement on the state of genetic testing for the channelopathies and cardiomyopathies: this document was developed as a partnership between the Heart Rhythm Society (HRS) and the European Heart Rhythm Association (EHRA). Europace 2011;13:1077-1109.

5. Hershberger RE, Kushner JD, Parks SB. In: Pagon RA, Bird TD, Dolan CR, Stephens K, Adam MP (eds). GeneReviews. University of Washington: Seattle, WA, 1993. http://www.ncbi.nlm. nih.gov/books/NBK1309/. Accessed 2013.

6. Biagini E, Coccolo F, Ferlito M, et al. Dilated-hypokinetic evolution of hypertrophic cardiomyopathy: prevalence, incidence, risk factors, and prognostic implications in pediatric and adult patients. J Am Coll Cardio/ 2005;46:1543-1550.

7. van der Zwaag PA, van Rijsingen IA, Asimaki A, et al. Phospholamban R14del mutation in patients diagnosed with dilated cardiomyopathy or arrhythmogenic right ventricular cardiomyopathy: evidence supporting the concept of arrhythmogenic cardiomyopathy. Eur J Heart Fail 2012;14:1199-1207.

8. Sen-Chowdhry S, Syrris P, Prasad SK, et al. Left-dominant arrhythmogenic cardiomyopathy: an under-recognized clinical entity. J Am Coll Cardiol 2008;52:2175-2187.

9. Posch MG, Posch MJ, Geier C, et al. A missense variant in desmoglein-2 predisposes to dilated cardiomyopathy. Mol Genet Metab 2008;95:74-80.

10. Elliott $P, O^{\prime}$ Mahony $C$, Syrris $P$, et al. Prevalence of desmosomal protein gene mutations in patients with dilated cardiomyopathy. Circ Cardiovasc Genet 2010;3:314-322.

11. Glöckle N, Kohl S, Mohr J, et al. Panel-based next generation sequencing as a reliable and efficient technique to detect mutations in unselected patients with retinal dystrophies. Eur J Hum Genet 2014;22:99-104.
12. Danzer M, Niklas N, Stabentheiner S, et al. Rapid, scalable and highly automated HLA genotyping using next-generation sequencing: a transition from research to diagnostics. BMC Genomics 2013;14:221.

13. Lemke JR, Riesch E, Scheurenbrand T, et al. Targeted next generation sequencing as a diagnostic tool in epileptic disorders. Epilepsia 2012;53:1387-1398.

14. NHLBI Grand Opportunity Exome Sequencing Project. https://esp. gs.washington.edu/drupal/. Accessed 2013.

15. Abecasis $G R$, Auton $A$, Brooks $L D$, et al. An integrated map of genetic variation from 1,092 human genomes. Nature 2012;491:56-65.

16. Norton N, Robertson PD, Rieder MJ, et al.; National Heart, Lung and Blood Institute GO Exome Sequencing Project. Evaluating pathogenicity of rare variants from dilated cardiomyopathy in the exome era. Circ Cardiovasc Genet 2012;5:167-174.

17. Kenna KP, McLaughlin RL, Hardiman O, Bradley DG. Using reference databases of genetic variation to evaluate the potential pathogenicity of candidate disease variants. Hum Mutat 2013;34:836-841.

18. Zimmerman RS, Cox S, Lakdawala NK, et al. A novel custom resequencing array for dilated cardiomyopathy. Genet Med 2010;12:268-278.

19. Li H, Durbin R. Fast and accurate long-read alignment with Burrows-Wheeler transform. Bioinformatics 2010;26:589-595.

20. McKenna A, Hanna M, Banks E, et al. The Genome Analysis Toolkit: a MapReduce framework for analyzing next-generation DNA sequencing data. Genome Res 2010;20:1297-1303.

21. DePristo MA, Banks E, Poplin R, et al. A framework for variation discovery and genotyping using next-generation DNA sequencing data. Nat Genet 2011;43:491-498.

22. Aronson SJ, Clark EH, Babb LJ, et al. The Genelnsight Suite: a platform to support laboratory and provider use of DNA-based genetic testing. Hum Mutat 2011;32:532-536.

23. Herman DS, Lam L, Taylor MR, et al. Truncations of titin causing dilated cardiomyopathy. N Engl J Med 2012;366:619-628.

24. Andreasen C, Nielsen JB, Refsgaard L, et al. New population-based exome data are questioning the pathogenicity of previously cardiomyopathy-associated genetic variants. Eur J Hum Genet 2013;21:918-928.

25. Jordan DM, Kiezun A, Baxter SM, et al. Development and validation of a computational method for assessment of missense variants in hypertrophic cardiomyopathy. Am J Hum Genet 2011;88:183-192.

26. Lakdawala NK, Funke BH, Baxter $S$, et al. Genetic testing for dilated cardiomyopathy in clinical practice. J Card Fail 2012;18:296-303.

27. Olson TM, Illenberger S, Kishimoto NY, Huttelmaier S, Keating MT, Jockusch BM. Metavinculin mutations alter actin interaction in dilated cardiomyopathy. Circulation 2002;105:431-437.

28. Vasile VC, Ommen SR, Edwards WD, Ackerman MJ. A missense mutation in a ubiquitously expressed protein, vinculin, confers susceptibility to hypertrophic cardiomyopathy. Biochem Biophys Res Commun 2006;345:998-1003.

29. Vasile VC, Will ML, Ommen SR, Edwards WD, Olson TM, Ackerman MJ. Identification of a metavinculin missense mutation, R975W, associated with both hypertrophic and dilated cardiomyopathy. Mol Genet Metab 2006;87:169-174.

30. Zemljic-Harpf AE, Miller JC, Henderson SA, et al. Cardiac-myocyte-specific excision of the vinculin gene disrupts cellular junctions, causing sudden death or dilated cardiomyopathy. Mol Cell Biol 2007;27:7522-7537.

31. Garcia-Pavia P, Syrris P, Salas C, et al. Desmosomal protein gene mutations in patients with idiopathic dilated cardiomyopathy undergoing cardiac transplantation: a clinicopathological study. Heart 2011;97: 1744-1752. 